\title{
Metabolic Profiling of Eccentric Exercise-Induced Muscle Damage in Human Urine
}

\author{
Hyun-Jun Jang ${ }^{1,2, \dagger}$, Jung Dae Lee ${ }^{3, \dagger}$, Hyun-Sik Jeon ${ }^{4}$, Ah-Ram Kim ${ }^{4,5}$, Suhkmann Kim ${ }^{6}$, \\ Ho-Seong Lee ${ }^{4}$ and Kyu-Bong Kim ${ }^{1}$ \\ ${ }^{1}$ College of Pharmacy, Dankook University, Cheonan, Korea \\ ${ }^{2}$ Department of Animal Biotechnology, Chonbuk National University, Jeonju, Korea \\ ${ }^{3}$ Division of Toxicology, College of Pharmacy, Sungkyunkwan University, Suwon, Korea \\ ${ }^{4}$ Department of Kinesiologic Medical Science, Graduate School, Dankook University, Cheonan, Korea \\ ${ }^{5}$ Department of Physical Therapy, Namseoul University, Cheonan, Korea \\ ${ }^{6}$ Department of Chemistry and Chemistry Institute of Functional Materials, Pusan National University, Busan, Korea
}

\begin{abstract}
Skeletal muscle can be ultrastructurally damaged by eccentric exercise, and the damage causes metabolic disruption in muscle. This study aimed to determine changes in the metabolomic patterns in urine and metabolomic markers in muscle damage after eccentric exercise. Five men and 6 women aged 19 23 years performed 30 min of the bench step exercise at 70 steps per min at a determined step height of $110 \%$ of the lower leg length, and stepping frequency at 15 cycles per min. ${ }^{1} \mathrm{H}$ NMR spectral analysis was performed in urine collected from all participants before and after eccentric exercise-induced muscle damage conventionally determined using a visual analogue scale (VAS) and maximal voluntary contraction (MVC). Urinary metabolic profiles were built by multivariate analysis of principal component analysis (PCA) and orthogonal partial least square-discriminant analysis (OPLS-DA) using SIMCA-P. From the OPLS-DA, men and women were separated $2 \mathrm{hr}$ after the eccentric exercise and the separated patterns were maintained or clarified until $96 \mathrm{hr}$ after the eccentric exercise. Subsequently, urinary metabolic profiles showed distinct trajectory patterns between men and women. Finally, we found increased urinary metabolites (men: alanine, asparagine, citrate, creatine phosphate, ethanol, formate, glucose, glycine, histidine, and lactate; women: adenine) after the eccentric exercise. These results could contribute to understanding metabolic responses following eccentric exercise-induced muscle damage in humans.
\end{abstract}

Key words: Metabolomic analysis, Skeletal muscle, Eccentric exercise, Sex-dependent patterns

\section{INTRODUCTION}

Exercise-induced muscle damage (EIMD) occurs by unaccustomed physical activity or rapid and excessive activity (1). EIMD is more commonly associated with eccentric exercise that lengthens the muscle under tension

Correspondence to: Kyu-Bong Kim, College of Pharmacy, Dankook University, 119 Dandae-ro, Cheonan, Chungnam 31116, Korea

E-mail: kyubong@dankook.ac.kr

Ho-Seong Lee, Department of Kinesiologic Medical Science, Graduate School, Dankook University, 119 Dandae-ro, Cheonan, Chungnam 31116, Korea

E-mail: hoseh28@dankook.ac.kr

${ }^{\dagger}$ The first two authors contributed equally to this work. rather than concentric exercise that shortens it (2). In the previous studies, eccentric contractions have shown to generate more powers (3) and decrease recruitment of motor units (4) than concentric contraction. In addition, it has suggested that eccentric action decreases attached cross-bridges as it increases the muscle fibre length (5).

This is an Open-Access article distributed under the terms of the Creative Commons Attribution Non-Commercial License (http:// creativecommons.org/licenses/by-nc/3.0) which permits unrestricted non-commercial use, distribution, and reproduction in any medium, provided the original work is properly cited. 
Therefore, eccentric contraction can more mechanically stress out the muscle than concentric contraction and the mechanical stress can contribute to inducing failure of febres (6).

EIMD physically causes stiffness, swelling, decreased force of muscular contraction, and delayed-onset muscular soreness (7), and it physiologically increases muscle blood flow, creatine kinase (CK) activity, lactate dehydrogenase, aldolase, aspartate transferase, and the circulating concentration of muscular cells such as skeletal troponin I, myoglobin and myosin in the blood plasma or serum (819). Unlike blood plasma or serum, there is no urinary CK standard because of the limitation of available information for exercise-induced CK increases and renal function (20). Instead, indexes of pigmenturia such as hematuria, hemoglobinuria and myoglobinuria are commonly applied to evaluate rhabdomyolysis in urine from EIMD. Clinically, these change appear from 24 to $48 \mathrm{hr}$ after exercise, and usually disappear after $72 \mathrm{hr}$ of rest (21). Additionally, EIMD based on urine could be evaluated by creatine/ creatinine ratio, collagen constituents including hydroxyproline and hydroxylysine, or uric acid (22). However, the urinary markers for EIMD are still limited because these indexes cover the phenotypes that include muscle diseases and renal failure (23).

EIMD-induced muscle is known to result in the release of cellular components through serial processes, beginning with depleting ATP, causing the leakage of extracellular calcium ions into intracellular space due to both $\mathrm{Na}$ $\mathrm{K}$-ATPase and $\mathrm{Ca}^{2+}$-ATPase pump dysfunction. The leakage of the contents of muscular cells into the circulation can increase intracellular proteolytic enzyme activity and promote muscle protein degradation and augmented cell permeability $(24,25)$. Especially, EIMD can be triggered by the loss of $\mathrm{Ca}^{2+}$ homeostasis and the initiation of the $\mathrm{Ca}^{2+}$ overload because of decreased action of $\mathrm{Ca}^{2+}$-adenosine triphosphatase (ATPase) during physical activity $(26,27)$. The disruption of cellular $\mathrm{Ca}^{2+}$ balance activates $\mathrm{Ca}^{2+}$ dependent proteolytic and phospholipolytic pathways which are related with the removal and repair of the damaged muscle $(28,29)$. These cellular responses are regarded as a series of complex events involving increased oxidative stress and inflammatory and immune responses $(6,30)$. However, the mechanisms and metabolic processes of EIMD are not entirely understood.

The omics is a systemic biology to understand the biological process for genomes, transcriptomes, proteomes, and metabolomes in organisms. Metabolomics of the omics is defined as the scientific study of biochemical processes involving endogenous metabolites and informs us about instantaneous and final responses to stresses in various organisms including humans $(31,32)$. Recently, these metabolomic approaches have been extended to sports science to identify biomarkers related to changes in physical performance, muscle fatigue, and muscle damage caused by exercise (33).

In this study, we tried to evaluate EIMD and trace its recovery pattern after eccentric exercise using urinary metabolites. Our results could contribute to understanding changes in the metabolomic patterns in urine and to identifying metabolomic markers in muscle damage after eccentric exercise.

\section{MATERIALS AND METHODS}

Subjects. Five male subjects (mean \pm standard deviation, age: $21.20 \pm 2.05$ years; weight: $73.38 \pm 7.52 \mathrm{~kg}$; height: $173.40 \pm 4.04 \mathrm{~cm}$; muscle: $34.86 \pm 1.90 \mathrm{~kg}$; body

Table 1. Physical properties including sex, age, height, body weight, muscle, body fat, and body mass index (BMI) of the individual subjects were recorded before the eccentric exercise

\begin{tabular}{llcccccc}
\hline \hline ID & Sex & Age (year) & Height $(\mathrm{cm})$ & Body weight $(\mathrm{kg})$ & Muscle $(\mathrm{kg})$ & Body fat $(\mathrm{kg})$ & Body mass index (BMI) \\
\hline Subject1 & Male & 22.00 & 168.00 & 67.20 & 34.30 & 11.50 & 23.00 \\
Subject2 & Male & 23.00 & 173.00 & 70.90 & 33.50 & 11.90 & 23.70 \\
Subject3 & Male & 19.00 & 172.00 & 67.20 & 33.10 & 13.50 & 22.70 \\
Subject4 & Male & 19.00 & 175.00 & 76.70 & 35.60 & 14.00 & 25.00 \\
Subject5 & Male & 23.00 & 179.00 & 84.90 & 37.80 & 22.30 & 26.50 \\
Mean & & 21.20 & 173.40 & 73.38 & 34.86 & 14.64 & 24.18 \\
SD & & 2.05 & 4.04 & 7.52 & 1.90 & 4.41 & 1.57 \\
Subject6 & Female & 21.00 & 170.00 & 56.60 & 24.70 & 19.80 & 21.60 \\
Subject7 & Female & 20.00 & 158.00 & 53.00 & 23.00 & 21.00 & 21.20 \\
Subject8 & Female & 21.00 & 164.00 & 53.60 & 22.90 & 22.40 & 2.90 \\
Subject9 & Female & 20.00 & 166.00 & 56.80 & 23.00 & 25.40 & 2.60 \\
Subject10 & Female & 20.00 & 158.00 & 60.70 & 23.30 & 18.20 & 2.30 \\
Subject11 & Female & 21.00 & 157.00 & 54.50 & 21.50 & 28.20 & 2.10 \\
Mean & & 20.50 & 162.17 & 55.87 & 23.07 & 22.50 & 1.73 \\
SD & & 0.55 & 5.31 & 2.83 & 1.02 & 3.71 & \\
\hline
\end{tabular}

SD, standard deviation. 
fat: $14.64 \pm 4.41 \mathrm{~kg}$; body mass index: $24.18 \pm 1.57)$ and 6 female subjects (mean \pm standard deviation; age: $20.50 \pm$ 0.55 years; weight: $55.87 \pm 2.83 \mathrm{~kg}$; height: $162.17 \pm 5.31$ $\mathrm{cm}$; muscle: $23.07 \pm 1.02 \mathrm{~kg}$; body fat: $22.50 \pm 3.71$; body mass index: $21.28 \pm 1.73)$ were included in the study. Individual information is shown in Table 1. All participants self-reported as being recreationally active (undertaking no more than $1 \mathrm{hr}$ of "moderate" physical activity per week) and did not take part in any structured resistance training. None of the female participants had ever used any form of estrogen-based contraception. All women reported regular menstrual cycles, documenting an average cycle length of $28 \pm 1$ days. Females were tested on the 14th day (selfreported) of the menstrual cycle to measure estrogen levels at ovulation (34). Exclusion criteria included any resistance training in the last six months, occupation or lifestyle that required regular heavy lifting or carrying, any known muscle disorder, the use of dietary supplements (i.e., vitamin E), and any musculoskeletal injury in the last three months. All inclusion and exclusion criteria were determined through participant questionnaire prior to inclusion within this study. This study was approved by the Ethics Committee of Dankook University, in accordance with the ethical standards of the Declaration of Helsinki (DKU 2015-10-005).

Eccentric exercise. To induce muscle damage by eccentric exercise, the subjects performed a single bout of 30 min of bench-stepping at 60 steps per min at a prede- termined step height of $110 \%$ of the lower leg length (35). Using the present exercise protocol, the exercise work rate can be determined using the mass lifted (body mass or body mass $+10 \mathrm{~kg})$ the step height $(110 \%$ of the lower leg length, on average $58 \mathrm{~cm})$, the gravitational constant and the stepping frequency (15 cycles per $\min$ ) (36).

Measuring muscle soreness. A visual analogue scale (VAS) was used to assess the volunteers' muscle soreness. In each evaluation, the same researcher instructed the subjects in a standardized manner to perform a sub-maximal voluntary isometric knee extensor contraction, marking a vertical line at the scale point that best reflected their muscle soreness. The 100-mm horizontal line of the VAS had no marks or numbers, only indications of no soreness at the beginning or extreme soreness at the end of the exercise line. The soreness was quantified using the distance between the initial point line $(0 \mathrm{~mm})$ and the point marked by the subject (37).

Measurement of muscle strength. Subjects were properly positioned with the dominant lower limb on the Isokinetic Dynamometer Biodex System 3 Pro (Biodex Medical System, Shirley, NY, USA), following the manufacturer's recommendations for evaluating knee flexion-extension movements. Before each evaluation, a warm-up was performed of 10 concentric knee flexion-extension repetitions at $180^{\circ} \mathrm{seg}^{-1}$ and maximal range of motion. Muscle strength was assessed through the highest torque value

(A)

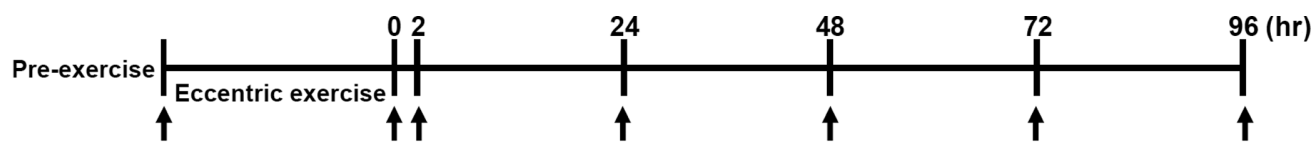

$\uparrow$ Urine collection and measurement of VAS and MVC
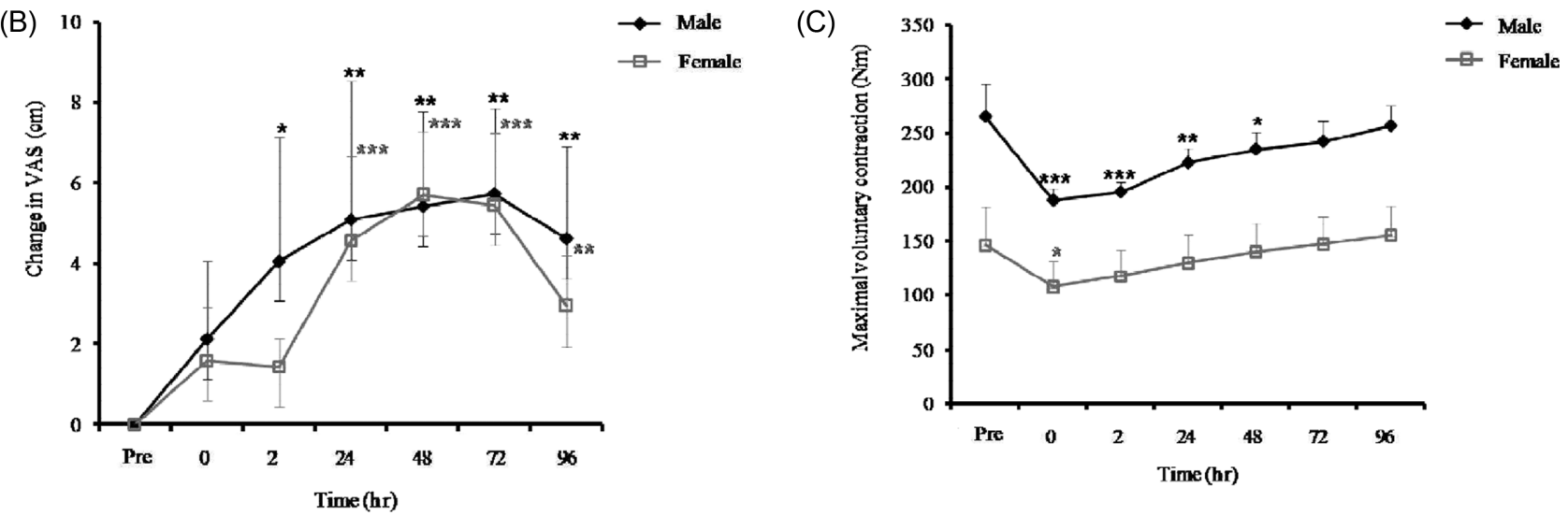

Fig. 1. (A) Schematic diagram of the experimental schedule in the study and (B) changes in visual analogue scale (VAS) and (C) maximal voluntary contraction (MVC) before the eccentric exercise (Pre) and according to the elapsed time (at $0,2,24,48,72$, and $96 \mathrm{hr}$ ) after the eccentric exercise. Values are mean \pm SD. ${ }^{*} p<0.05,{ }^{* *} p<0.01,{ }^{* * *} p<0.001$. 
obtained among three 5-s maximal voluntary contraction (MVC) at $60^{\circ}$ of knee flexion $\left(0^{\circ}=\right.$ full knee extension $)$. Participants received a two-min rest between each MVC to minimize any possible fatigue effects. Volunteers were previously instructed to perform maximal force and verbal encouragement was given by researchers in each MVC (38).

Urine collection. Urine was collected from the subjects before the exercise and again immediately, $2 \mathrm{hr}$, $24 \mathrm{hr}, 48 \mathrm{hr}, 72 \mathrm{hr}$, and $96 \mathrm{hr}$ after the exercise (Fig. 1A). The urine samples were stored at $-70^{\circ} \mathrm{C}$ until analysis.

${ }^{1} \boldsymbol{H}$ NMR spectroscopic analysis. After the urine samples were thawed at $4^{\circ} \mathrm{C}$, they were centrifuged to remove solids. A $600 \mu \mathrm{L}$ aliquot of the supernatant was added to a microcentrifuge tube containing $70 \mu \mathrm{L}$ of $\mathrm{D}_{2} \mathrm{O}$ solution with $5 \mathrm{mM}$ DSS and $10 \mathrm{mM}$ imidazole. DSS was used as the qualitative standard for the chemical shift scale. In addition, $30 \mu \mathrm{L}$ of $0.42 \%$ sodium azide was added. After vortexing, this solution was adjusted to $\mathrm{pH} 6.8$, and the urine sample was analyzed with a nuclear magnetic resonance (NMR) spectrometer within $48 \mathrm{hr}$. All spectra were determined using a Varian Unity Inova $600 \mathrm{MHz}$ spectrometer at Pusan National University (Busan, Korea) operating at $26^{\circ} \mathrm{C}$. One-dimensional NMR spectra were acquired with the following acquisition parameters: spectral width $24038.5 \mathrm{~Hz}, 12.53 \mathrm{~min}$ acquisition time, and $128 \mathrm{nt}$. Additional conditions were set of a relaxation delay time of $1 \mathrm{~s}$ and saturation power of 4 to suppress massive water peaks. The NMR spectra were reduced to data using the Chenomx NMR Suit program (ver. 4.6, Chenomx Inc., Edmonton, Alberta, Canada). The $\delta 0.0 \sim 10.0$ spectral region was segmented into regions of $0.04 \mathrm{ppm}$ width to provide 250 integrated regions in each NMR spectrum. This binning process endowed each segment with an integral value that provided an intensity distribution of the whole spectrum with 250 variables prior to the pattern recognition analysis. The spectrum region of water $(\delta 4.5 \sim$ 5.0) was removed from the analysis to prevent variation in water suppression efficiency. We also identified and quantified the spectra using the Chenomx NMR Suit Professional software package ver. 4.6 (Chenomx Inc.). DSS was used as the concentration reference at a concentration of $0.5 \mathrm{mM}$, and 2D NMR analysis was also performed to validate the identification of endogenous metabolites. Metabolite concentrations were expressed as relative ratio values normalized to creatinine concentration, assuming a constant rate of creatinine excretion in every urine sample.

Principal component analysis (PCA) and orthogonal partial least square-discriminant analysis (OPLS-DA). All data were converted from the NMR software format into Microsoft Excel (Microsoft, Seattle, WA, USA). Onedimensional NMR spectra data were imported into SIMCA-P (version 12.0, Umetrics Inc., Kinnelon, NJ, USA) for multivariate statistical analysis to examine intrinsic variations in the data set. These data were scaled using centered scaling prior to the PCA and OPLS-DA. For the scaling process, the average value of each variable was calculated and then subtracted from the data. PCA and OPLS-DA score plots were used to interpret intrinsic variation in the data.

Statistical analyses. The changes in the muscle soreness and MVC over time after the eccentric exercise were analyzed by a one-way repeated measures ANOVA, followed by a Tukey's post hoc test when a significant time effect was found to locate the time points that were different from the baseline values. All analyses were conducted with SPSS software (version 20.0, SPSS Inc., Chicago, IL, USA), and a $p<0.05$ was considered statistically significant. Means and standard deviations of the metabolites were also calculated using Microsoft Excel. The statistical significance $(p<0.05, p<0.01$, or $p<0.001)$ of apparent differences in metabolite concentrations between before and after the eccentric exercise were assessed using analysis of variance, followed by Bartlett's test (Prism 5.01, GraphPad, San Diego, CA, USA).

\section{RESULTS}

Muscle soreness developed after the eccentric exercise, peaking at 48 to $72 \mathrm{hr}$ later in both male and female subjects (Fig. 1B). The MVC torque decreased at 0 to $48 \mathrm{hr}$ after the eccentric exercise in the male subjects and $2 \mathrm{hr}$ later among the female subjects (Fig. 1C). However, the MVC torque had returned to baseline by $96 \mathrm{hr}$ after the eccentric exercise in both male and female subjects (Fig. 1C).

Through the metabolite analysis for the urine samples, the PCA showed clear separation of the metabolic patterns between male and female subjects at all time points (Fig. 2A). Subsequently, the trajectory analysis of PCA score plots for the metabolic patterns between male and female subjects can really reflect the sexual differences from muscle damages to its recovery. From the trajectory analysis, the metabolomic patterns of male subjects had changed rapidly from 24 to $72 \mathrm{hr}$ after the eccentric exercise and then gradually restored themselves by $96 \mathrm{hr}$. However, the metabolomic patterns of female subjects had changed conspicuously at only $0 \mathrm{hr}$ after the eccentric exercise and did not show the restoration that was seen among the males (Fig. 2B). When OPLS-DA was performed on the metabolites, metabolomic patterns in the male and female subjects were clearly separated at all time points (Fig. 2C).

To evaluate metabolites which contribute to metabolomic patterns for each group, variable importance plots (VIPs) were derived from the OPLS-DA between male 
(A)

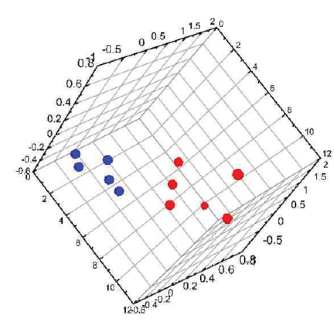

$48 \mathrm{hr}$

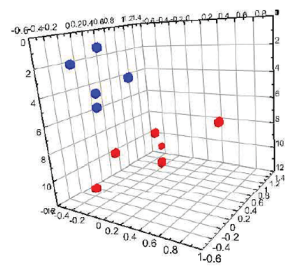

$0 \mathrm{hr}$

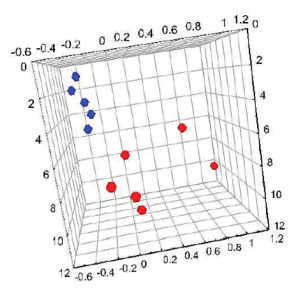

$72 \mathrm{hr}$

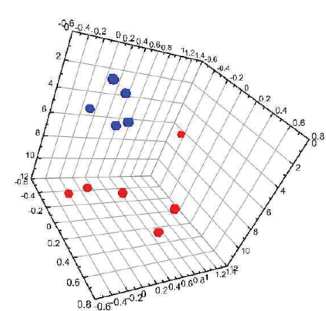

$2 \mathrm{hr}$

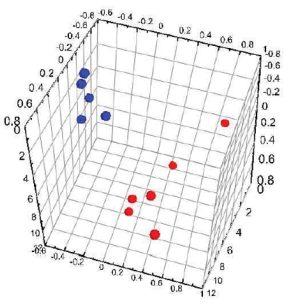

$96 \mathrm{hr}$

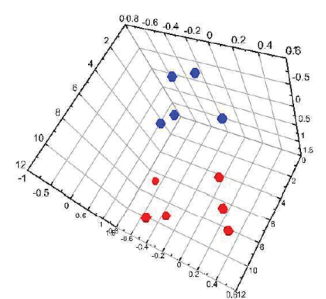

$24 \mathrm{hr}$

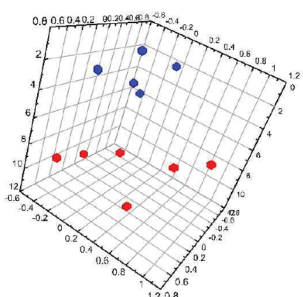

(B)

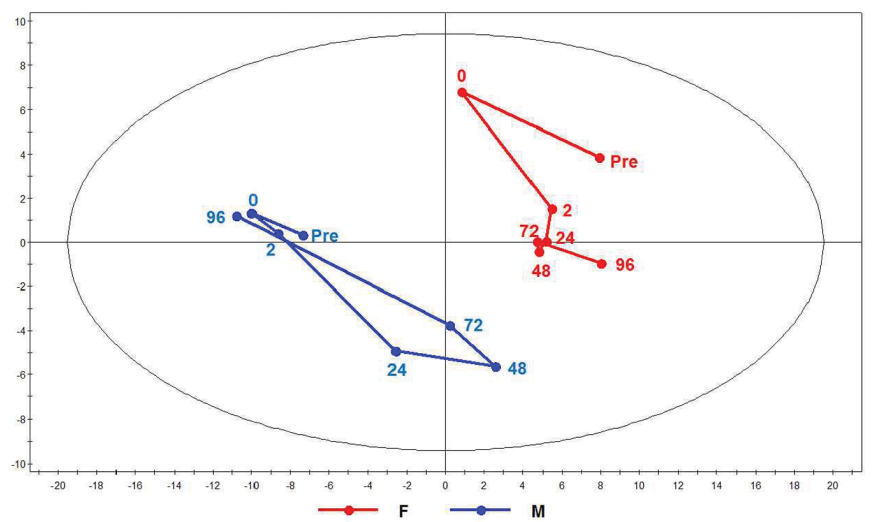

(C)

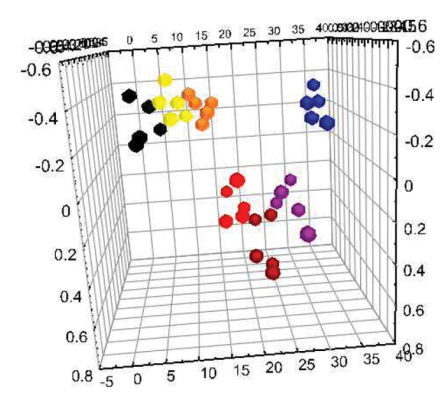

Male

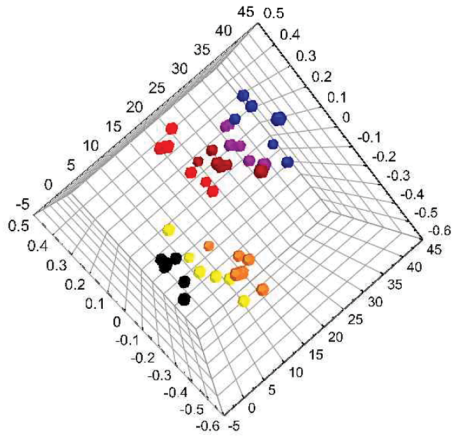

Female

ohr

$2 \mathrm{hr}$

$24 \mathrm{hr}$

$48 \mathrm{hr}$

$72 \mathrm{hr}$

$96 \mathrm{hr}$

Fig. 2. (A) Comparisons of metabolic patterns between male and female subjects according to the elapsed time after the eccentric exercise. Principal Components Analysis (PCA) before the eccentric exercise (Pre) $\left(R^{2} X: 0.653 ; Q^{2}: 0.0614\right)$ and PCA at $0 \mathrm{hr}$ (immediately after the eccentric exercise) ( $\left.R^{2} X: 0.608 ; Q^{2}: 0.107\right)$, at $2 \mathrm{hr}\left(R^{2} X: 0.61 ; Q^{2}:-0.0429\right), 24 \mathrm{hr}\left(R^{2} X: 0.58 ; Q^{2}: 0.0539\right), 48 \mathrm{hr}\left(R^{2} X:\right.$ $\left.0.563 ; Q^{2}:-0.141\right), 72 \mathrm{hr}\left(R^{2} X: 0.506 ; Q^{2}:-0.21\right)$, and $96 \mathrm{hr}\left(R^{2} X: 0.665 ; Q^{2}: 0.188\right)$ after the eccentric exercise, compared between males and females, respectively. (B) Trajectory analysis using the principal components analysis (PCA) $\left(R^{2} X: 0.681 ; Q^{2}: 0.547\right)$ based on mean data for ${ }^{1} \mathrm{H}$ NMR of urine samples of the subjects according to elapsed time (at $0,2,24,48,72$, and $96 \mathrm{hr}$ ) after the eccentric exercise. (C) The score plots showing that the clustering are completely separated in male $\left(R^{2} X: 0.551 ; R^{2} Y: 0.191 ; Q^{2}:-0.0411\right)$ and female $\left(R^{2} X: 0.483 ; R^{2} Y: 0.158 ; Q^{2}:-0.192\right)$ using the Orthogonal Partial Least Square-Discriminant Analysis (OPLS-DA). 
Table 2. VIP scores showed that list of metabolites which contributed to separation of the clustering in male $\left(R^{2} X: 0.551 ; R^{2} Y\right.$ : $\left.0.191 ; Q^{2}:-0.0411\right)$ and female $\left(R^{2} X: 0.483 ; R^{2} Y: 0.158 ; Q^{2}:-0.192\right)$ by OPLS-DA

\begin{tabular}{|c|c|c|c|c|c|}
\hline \multicolumn{3}{|c|}{ Male } & \multicolumn{3}{|c|}{ Female } \\
\hline Var ID (Primary) & Male.VIP[1] & $\begin{array}{c}1.89456 \\
\text { Male.VIP[1]cvSE }\end{array}$ & Var ID (Primary) & Female.VIP[1] & $\begin{array}{c}1.89456 \\
\text { Female.VIP[1]cvSE }\end{array}$ \\
\hline Glycine & 4.571 & 0.761416 & Glycine & 4.62347 & 4.53764 \\
\hline Citrate & 3.23447 & 1.35125 & Hippurate & 3.81074 & 2.41106 \\
\hline Formate & 2.13353 & 0.717861 & Taurine & 2.67864 & 6.68557 \\
\hline Creatine phosphate & 2.11266 & 1.64716 & Histidine & 2.65182 & 5.56657 \\
\hline Alanine & 1.99812 & 0.587374 & Adenine & 2.64272 & 4.37275 \\
\hline Guanidoacetate & 1.92338 & 1.63802 & Ethanolamine & 1.90545 & 2.09334 \\
\hline Trimethylamine N-oxide & 1.79513 & 2.1608 & Creatine phosphate & 1.8096 & 3.79375 \\
\hline Histidine & 1.72022 & 1.37944 & Citrate & 1.62474 & 8.11127 \\
\hline Taurine & 1.67516 & 1.49017 & cis-Aconitate & 1.14701 & 0.80235 \\
\hline Ethylene glycol & 1.45479 & 0.546222 & Formate & 0.988822 & 2.45472 \\
\hline Lactate & 1.23411 & 0.53149 & Dimethylamine & 0.951277 & 1.99825 \\
\hline Glutamine & 1.17523 & 0.857112 & Ethanol & 0.90434 & 0.993288 \\
\hline Glucose & 1.1471 & 0.39031 & Glucose & 0.834976 & 2.38004 \\
\hline Asparagine & 1.13309 & 0.735665 & Lactate & 0.789613 & 2.56846 \\
\hline Ethanol & 1.05674 & 0.422716 & Alanine & 0.787954 & 1.05048 \\
\hline Arginine & 0.970961 & 0.626348 & Trimethylamine N-oxide & 0.787139 & 5.26624 \\
\hline Adenine & 0.902511 & 0.662068 & 3-Hydroxyisovalerate & 0.770134 & 0.771259 \\
\hline Glycerol & 0.851086 & 0.462209 & Propylene glycol & 0.764305 & 1.44092 \\
\hline N,N-Dimethylglycine & 0.836798 & 0.282404 & Dimethyl sulfone & 0.757325 & 0.716034 \\
\hline Trigonelline & 0.819964 & 0.686261 & Methylmalonate & 0.755935 & 1.13527 \\
\hline Acetate & 0.817444 & 0.280176 & Lactose & 0.67113 & 1.44531 \\
\hline Dimethyl sulfone & 0.799348 & 0.448651 & 3-Indoxylsulfate & 0.643779 & 0.992767 \\
\hline Malonate & 0.777858 & 0.727546 & Xylose & 0.638257 & 1.18835 \\
\hline Trimethylamine & 0.765254 & 0.379615 & N6-Acetyllysine & 0.637389 & 0.650881 \\
\hline Lactose & 0.72369 & 0.515311 & 1-Methylnicotinamide & 0.635111 & 1.28124 \\
\hline Xylose & 0.71229 & 0.695227 & Trigonelline & 0.566073 & 1.59381 \\
\hline Methanol & 0.697198 & 0.199636 & 3-Hydroxybutyrate & 0.507793 & 0.909552 \\
\hline 4-Aminobutyrate & 0.676836 & 0.506111 & Malonate & 0.499509 & 1.15068 \\
\hline 3-Indoxylsulfate & 0.669647 & 0.762088 & Thymol & 0.4972 & 0.976628 \\
\hline Propylene glycol & 0.655221 & 0.465205 & Glutamate & 0.478324 & 0.547958 \\
\hline Isobutyrate & 0.633856 & 0.08158 & N,N-Dimethylglycine & 0.460773 & 0.609594 \\
\hline Anserine & 0.633435 & 0.151005 & N-Nitrosodimethylamine & 0.438651 & 2.31928 \\
\hline Aspartate & 0.621186 & 0.627908 & 4-Hydroxybenzoate & 0.433857 & 0.902987 \\
\hline N-Nitrosodimethylamine & 0.583811 & 0.567109 & Succinylacetone & 0.419752 & 0.521925 \\
\hline Creatine & 0.578945 & 0.598633 & 2-Hydroxyphenylacetate & 0.41534 & 0.742998 \\
\hline 3-Hydroxyisovalerate & 0.569219 & 0.392871 & Succinate & 0.387387 & 0.797767 \\
\hline $\begin{array}{l}\text { Glutaric acid } \\
\text { monomethyl ester }\end{array}$ & 0.566226 & 0.289886 & Trimethylamine & 0.368037 & 0.644605 \\
\hline Valine & 0.552121 & 0.175912 & Glutamine & 0.365716 & 1.09904 \\
\hline N6-Acetyllysine & 0.504685 & 0.167541 & Creatine & 0.362578 & 2.06171 \\
\hline 2-Hydroxy-3-methylvalerate & 0.496476 & 0.164713 & Aspartate & 0.352493 & 1.07166 \\
\hline 1-Methylnicotinamide & 0.494685 & 0.392253 & Betaine & 0.335485 & 2.18197 \\
\hline Acetone & 0.491547 & 0.206371 & 1,3-Dimethylurate & 0.332024 & 1.22418 \\
\hline 2-Oxoglutarate & 0.472701 & 0.509611 & Methionine & 0.327067 & 0.488806 \\
\hline Ethanolamine & 0.462338 & 1.06293 & Histamine & 0.308111 & 1.28022 \\
\hline 3-Methyl-2-oxovalerate & 0.445005 & 0.238199 & Valine & 0.299257 & 0.582553 \\
\hline 2-Octenoate & 0.424973 & 0.389413 & Uracil & 0.283379 & 0.710501 \\
\hline trans-Aconitate & 0.423043 & 0.556248 & Arginine & 0.280329 & 1.10447 \\
\hline Choline & 0.415968 & 0.218118 & 4-Aminobutyrate & 0.269595 & 1.09926 \\
\hline cis-Aconitate & 0.407804 & 0.55756 & Asparagine & 0.253733 & 0.725486 \\
\hline Betaine & 0.398263 & 0.887558 & $\begin{array}{l}\text { Glutaric acid } \\
\text { monomethyl ester }\end{array}$ & 0.24793 & 0.491761 \\
\hline
\end{tabular}


Table 2. Continued

\begin{tabular}{|c|c|c|c|c|c|}
\hline \multicolumn{3}{|c|}{ Male } & \multicolumn{3}{|c|}{ Female } \\
\hline Var ID (Primary) & Male.VIP[1] & $\begin{array}{c}1.89456 \\
\text { Male.VIP[1]cvSE }\end{array}$ & Var ID (Primary) & Female.VIP[1] & $\begin{array}{c}1.89456 \\
\text { Female.VIP[1]cvSE }\end{array}$ \\
\hline 2-Hydroxybutyrate & 0.351128 & 0.294334 & Methanol & 0.238896 & 0.379045 \\
\hline Methionine & 0.331838 & 0.538283 & Leucine & 0.231152 & 0.483716 \\
\hline 1,3-Dimethylurate & 0.323249 & 0.235596 & Choline & 0.221929 & 0.439599 \\
\hline Glutarate & 0.316202 & 0.418292 & Pyruvate & 0.211881 & 0.941236 \\
\hline Acetoacetate & 0.313934 & 0.481351 & $\begin{array}{l}\text { 4-Hydroxy-3- } \\
\text { methoxymandelate }\end{array}$ & 0.207803 & 0.61239 \\
\hline 1,3-Dihydroxyacetone & 0.309266 & 0.183771 & N-Phenylacetylglycine & 0.199173 & 1.17539 \\
\hline N-Phenylacetylglycine & 0.309063 & 0.811053 & 2-Hydroxyisobutyrate & 0.19341 & 0.483164 \\
\hline 2-Oxoisocaproate & 0.305994 & 0.113296 & 3-Phenyllactate & 0.185375 & 0.63094 \\
\hline Leucine & 0.296766 & 0.435264 & N-Acetylglucosamine & 0.176375 & 0.593308 \\
\hline Glutamate & 0.293844 & 0.86197 & Cytidine & 0.158637 & 0.403555 \\
\hline Epicatechin & 0.292327 & 0.116411 & Isobutyrate & 0.151427 & 0.541375 \\
\hline Xanthosine & 0.2846 & 0.127379 & 2-Hydroxybutyrate & 0.145103 & 1.04791 \\
\hline Succinylacetone & 0.271647 & 0.350153 & Guanidoacetate & 0.129694 & 2.71269 \\
\hline Thymol & 0.254143 & 0.323742 & Epicatechin & 0.128747 & 0.282884 \\
\hline Succinate & 0.246905 & 0.304167 & Xanthosine & 0.125117 & 0.316036 \\
\hline Acetylsalicylate & 0.236725 & 0.424659 & Anserine & 0.113301 & 0.458756 \\
\hline Pyruvate & 0.199842 & 0.297075 & 2-Oxoglutarate & 0.113046 & 0.558352 \\
\hline 4-Hydroxybenzoate & 0.18721 & 0.277188 & Uridine & 0.09736 & 0.39164 \\
\hline 2-Hydroxyphenylacetate & 0.18137 & 0.122769 & Acetoacetate & 0.096019 & 0.524509 \\
\hline Histamine & 0.176062 & 0.73845 & 2-Octenoate & 0.092254 & 0.589411 \\
\hline Methylmalonate & 0.162552 & 0.489295 & Glutarate & 0.087717 & 0.924993 \\
\hline Uridine & 0.159729 & 0.175195 & Carnitine & 0.087113 & 0.662933 \\
\hline N-Acetylglucosamine & 0.156865 & 0.21566 & $\begin{array}{l}\text { 2-Hydroxy-3- } \\
\text { methylvalerate }\end{array}$ & 0.076218 & 0.777415 \\
\hline $\begin{array}{l}\text { 4-Hydroxy-3- } \\
\text { methoxymandelate }\end{array}$ & 0.15036 & 0.216263 & Ethylene glycol & 0.049435 & 1.09843 \\
\hline Uracil & 0.138542 & 0.513323 & 1,3-Dihydroxyacetone & 0.038835 & 0.125192 \\
\hline Cytidine & 0.093631 & 0.210719 & 3-Methyl-2-oxovalerate & 0.0363 & 0.423631 \\
\hline 3-Phenyllactate & 0.090093 & 0.440311 & Acetate & 0.031847 & 0.547257 \\
\hline Hippurate & 0.088477 & 0.852423 & 2-Oxoisocaproate & 0.031675 & 0.367915 \\
\hline Carnitine & 0.081513 & 0.355035 & trans-Aconitate & 0.027163 & 0.679173 \\
\hline 2-Hydroxyisobutyrate & 0.056391 & 0.169117 & Acetone & 0.023618 & 0.385497 \\
\hline Dimethylamine & 0.048648 & 0.689478 & Glycerol & 0.023313 & 0.720811 \\
\hline 3-Hydroxybutyrate & 0.029112 & 0.16385 & Acetylsalicylate & 0.013139 & 0.32408 \\
\hline
\end{tabular}

VIP, variable important plots.

Bold box indicates major metabolites which have more than VIP score 1 by the OPLS-DA.

Table 3. List of sex-specific and common metabolites from the major metabolites (VIP scores $>1$ ) in male and female

\begin{tabular}{lcl}
\hline \hline Clustering & Total & Urinary metabolites \\
\hline Male only & 10 & Alanine, asparagine, ethanol, ethylene glycol, formate, glucose, \\
glutamine, guanidoacetate, lactate, trimethylamine n-oxide \\
Female only & 4 & Adenine, cis-aconitate, ethanolamine, hippurate \\
Overlap between male and female & 5 & Citrate, creatine phosphate, glycine, histidine, taurine \\
\hline
\end{tabular}

and female subjects. Total 82 metabolites were scored to reflect their importance for the metabolomic patterns between male and female subjects (Table 2). When VIP scores were confined to $>1$ from the VIPs to select more convincing metabolites, 10 and 4 metabolites were found in males and females and 5 metabolites were commonly found in both males and females (Table 3).

From the statistical analysis, 10 of the selected 15 metabolites in male subjects had increased significantly after the eccentric exercise, most between 24 and $72 \mathrm{hr}$ later. Alanine, formate, and glycine had commonly increased between $24 \mathrm{hr}$ and $72 \mathrm{hr}$, whereas asparagine, citrate, etha- 
(A)
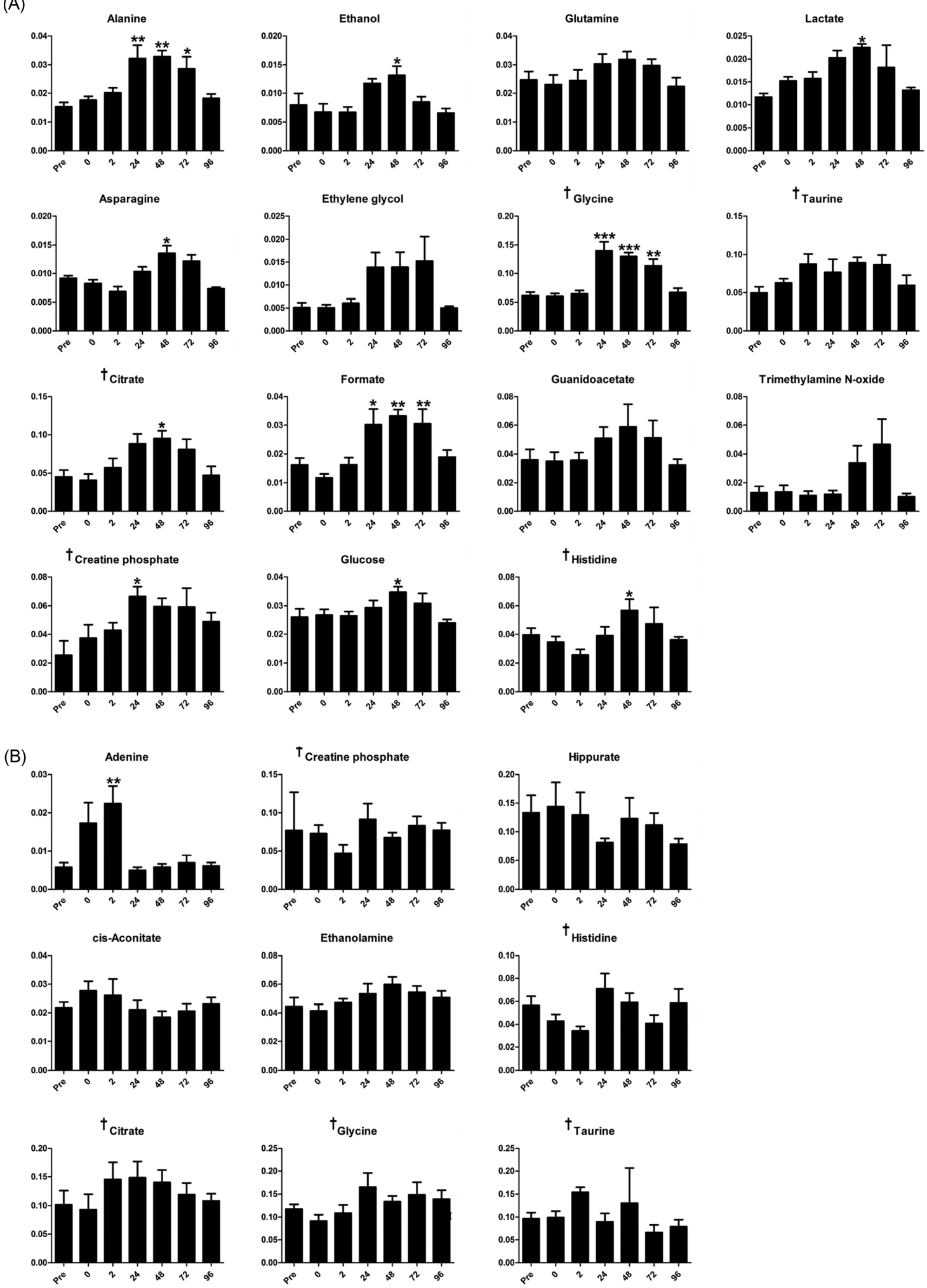

Fig. 3. (A) From the male VIP scores, concentration of the major metabolites (VIP $>1$ ) in urine samples of the subjects before the eccentric exercise (Pre) and according to the elapsed time (at 0, 2, 24, 48, 72, and $96 \mathrm{hr}$ ) after the eccentric exercise. (B) From the female VIP scores, concentration of the major metabolites (VIP $>1$ ) in urine samples of the subjects before the eccentric exercise (Pre) and according to the elapsed time (at 0, 2, 24, 48, 72, and $96 \mathrm{hr}$ ) after the eccentric exercise. Error bars are expressed as SD. ${ }^{*} p<0.05 ;{ }^{* *} p<0.01 ;{ }^{* * *} p<0.001$. Indicates overlapped metabolites among the major metabolites of male and female. 
nol, glucose, histidine, and lactate only increased $48 \mathrm{hr}$ later. In addition, creatine phosphate increased $24 \mathrm{hr}$ later (Fig. 3A). In contrast, among the females, only adenine from the selected 9 metabolites had increased significantly $2 \mathrm{hr}$ after the eccentric exercise (Fig. 3B).

\section{DISCUSSION}

Exercise-induced muscle damage that is closely related with eccentric exercise leads to increased intramuscular proteins in the blood (e.g., creatine kinase) (39) and the delayed onset of muscle soreness (40) but decreased muscle strength and range of motion (41). Researchers in previous studies have measured VAS and MVC to assess muscle soreness and muscle strength, respectively $(38,42)$. In the current study, muscle soreness in both males and females increased after the eccentric exercise and distinctly decreased beginning $72 \mathrm{hr}$ later, and the patterns were similar between the men and the women (Fig. 1B). In addition, male MVC torque decreased rapidly at $0 \mathrm{hr}$ after the eccentric exercise and steadily recovered from 2 to $96 \mathrm{hr}$ later, and female MVC torque decreased only at $0 \mathrm{hr}$ after the exercise $(p<0.05)$; however the recovery patterns were similar in both groups (Fig. 1C). These results suggested that the subjects responded to EIMD in a sex-dependent manner.

Notably, PCA metabolomic pattern results also differed between males and females without the eccentric exercise (Fig. 2A). A number of studies have shown sex differences in muscle damage and repair processes (30). Other studies, however, have reported that these sex-different responses to muscle-damaging exercise are related to poor study design and that there may be little or no difference between males and females in the responses under elaborated conditions $(43,44)$. Furthermore, the previous metabolomic research has shown distinct baseline metabolomic patterns between males and females $(45,46)$, and the hormone fluctuations related to menstruation could directly or indirectly affect responses to exercise-induced muscle disruption (47). In our results, the metabolomic patterns among the females were more dispersed than were the metabolomic patterns in the men (Fig. 2A). Collectively, the differences in the metabolomic patterns between the male and female subjects suggest origins in innate sex differences rather than effects from muscle damage.

In the metabolomic trajectory pattern using PCA, the mean pre-exercise values among the men shifted rapidly at $24 \mathrm{hr}$ and reached their maximums at $48 \mathrm{hr}$ after the eccentric exercise, appearing to then return to the preexercise values by $96 \mathrm{hr}$ later. In contrast, the shift in the female mean reached its maximum during pre-exercise, following which the women's shift patterns were more erratic than those of the men (Fig. 2B). From these results, we suggest that metabolic changes in men following mus- cle damage reach their maximum at $48 \mathrm{hr}$ after eccentric exercise and that these changes stabilize to their pre-exercise values more slowly than do conventional VAS and MVC.

In metabolomic approaches, creatine kinase and the related metabolites such as creatine and creatine phosphate have been widely considered biomarkers for muscle damage (30). The creatine phosphate circuit, which shows the rephosphorylation of creatine in mitochondria using ATP, together form the core of an energy network in both the cytosol and mitochondria of tissues that demand high energy such as the muscles and the brain (48). In addition, muscles take in glucose from the circulating blood to produce energy, and the glucose in the muscles degrades to pyruvate through glycolysis. Subsequently, pyruvate generates acetyl-CoA to form citrate through the tricarboxylic acid cycle or is converted to alanine or lactate through serial processes. Recently, citrate synthase activity was validated as a biomarker for mitochondrial density in skeletal muscle (49). Meanwhile, the transmitted alanine and lactate are transferred from muscles to liver by blood circulation to resynthesize glucose. From the energy metabolism processes, alanine and lactate increase in the body's circulation system (50). Thus, alanine, citrate, creatine phosphate, glucose, and lactate could be evaluated as markers that are correlated with energy production after energy consumption by exercise.

Intensive and prolonged exercise has also been correlated with the generation of free radicals and with oxidative damage to cellular constituents $(51,52)$. One study showed that histidine in microbes could be utilized as a source of antioxidant to cells (53). This result implies that histidine in the body could contribute to relieving oxidative stress from exercise. Muscle damage also involves inflammatory responses in the body (54). Previously, glycine infusion has been shown to have a cytoprotective effect against ischemia-reperfusion injury $(55,56)$, and recent studies have indicated that glycine could be an effective anti-inflammatory agent that preserves muscle function $(57,58)$. Therefore, histidine and glycine could be considered indirect EIMD markers.

Asparagine, ethanol, and formate were also increased after the eccentric exercise. Formate and ethanol in microbes have in particular been reported for anaerobic energy production, including glucose metabolism under anaerobic conditions or fermentation after pyruvate formation (5961). However, the roles of these metabolites after EIMD are still unclear.

In conclusion, we found changes in urinary metabolomic patterns after participation in eccentric exercise and increased levels of specific metabolites from 24 to $72 \mathrm{hr}$, which suggested involvement in muscle damage or recovery. These results also suggested that the endogenous metabolites increased more among men following EIMD 
and that further study is needed among females in order to understand possible interferential effects of female hormones such as estrogen.

\section{ACKNOWLEDGMENTS}

This work was supported by a National Research Foundation of Korea (NRF) grant (NRF-2011-0013659 and NRF-2017R1A2B4004758) funded by the Korea government (MEST). No conflicts of interests, financial, or otherwise are declared by the authors.

Received January 30, 2018; Revised April 29, 2018; Accepted May 14, 2018

\section{REFERENCES}

1. Stauber, W.T. (1989) Eccentric action of muscles: physiology, injury, and adaptation. Exerc. Sport Sci. Rev., 17, 157185.

2. Brown, S., Day, S. and Donnelly, A. (1999) Indirect evidence of human skeletal muscle damage and collagen breakdown after eccentric muscle actions. J. Sports Sci., 17, $397-$ 402.

3. Woledge, R.C., Curtin, N.A. and Homsher, E. (1985) Energetic aspects of muscle contraction. Monogr. Physiol. Soc., 41, 1-357.

4. Bigland-Ritchie, B. and Woods, J.J. (1974) Integrated EMG and oxygen uptake during dynamic contractions of human muscles. J. Appl. Physiol., 36, 475-479.

5. McCully, K.K. and Faulkner, J.A. (1986) Characteristics of lengthening contractions associated with injury to skeletal muscle fibers. J. Appl. Physiol., 61, 293-299.

6. Tee, J.C., Bosch, A.N. and Lambert, M.I. (2007) Metabolic consequences of exercise-induced muscle damage. Sports Med., 37, 827-836.

7. Byrne, C., Twist, C. and Eston, R. (2004) Neuromuscular function after exercise-induced muscle damage: theoretical and applied implications. Sports Med., 34, 49-69.

8. Asp, S., Daugaard, J.R. and Richter, E.A. (1995) Eccentric exercise decreases glucose transporter GLUT4 protein in human skeletal muscle. J. Physiol., 482, 705-712.

9. Asp, S., Rohde, T. and Richter, E.A. (1997) Impaired muscle glycogen resynthesis after a marathon is not caused by decreased muscle GLUT-4 content. J. Appl. Physiol., 83, 1482-1485.

10. Asp, S., Daugaard, J.R., Kristiansen, S., Kiens, B., Richter, E.A. (1998) Exercise metabolism in human skeletal muscle exposed to prior eccentric exercise. J. Physiol., 509, 305313.

11. Asp, S., Daugaard, J.R., Rohde, T., Adamo, K. and Graham, T. (1999) Muscle glycogen accumulation after a marathon: roles of fiber type and pro- and macroglycogen. J. Appl. Physiol., 86, 474-478.

12. Costill, D.L., Pascoe, D.D., Fink, W.J., Robergs, R.A., Barr, S.I. and Pearson, D. (1990) Impaired muscle glycogen resynthesis after eccentric exercise. J. Appl. Physiol., 69, 46-50.

13. Evans, W.J., Meredith, C.N., Cannon, J.G., Dinarello, C.A.,
Frontera, W.R., Hughes, V.A., Jones, B.H. and Knuttgen, H.G. (1986) Metabolic changes following eccentric exercise in trained and untrained men. J. Appl. Physiol., 61, 18641868.

14. Kirwan, J.P., Hickner, R.C., Yarasheski, K.E., Kohrt, W.M., Wiethop, B.V. and Holloszy, J.O. (1992) Eccentric exercise induces transient insulin resistance in healthy individuals. $J$. Appl. Physiol., 72, 2197-2202.

15. Nosaka, K. and Clarkson, P.M. (1995) Muscle damage following repeated bouts of high force eccentric exercise. Med. Sci. Sports Exerc., 27, 1263-1269.

16. Selkow, N.M., Day, C., Liu, Z., Hart, J.M., Hertel, J. and Saliba, S.A. (2012) Microvascular perfusion and intramuscular temperature of the calf during cooling. Med. Sci. Sports Exerc., 44, 850-856.

17. Semark, A., Noakes, T.D., St Clair, G.A. and Lambert, M.I. (1999) The effect of a prophylactic dose of flurbiprofen on muscle soreness and sprinting performance in trained subjects. J. Sports Sci., 17, 197-203.

18. Sorichter, S., Puschendorf, B. and Mair, J. (1999) Skeletal muscle injury induced by eccentric muscle action: muscle proteins as markers of muscle fiber injury. Exerc. Immunol. Rev., 5, 5-21.

19. Tuominen, J.A., Ebeling, P., Bourey, R., Koranyi, L., Lamminen, A., Rapola, J., Sane, T., Vuorinen-Markkola, H. and Koivisto, V.A. (1996) Postmarathon paradox: insulin resistance in the face of glycogen depletion. Am. J. Physiol., 270, E336-E343.

20. Brancaccio, P., Lippi, G. and Maffulli, N. (2010) Biochemical markers of muscular damage. Clin. Chem. Lab. Med., 48, 757-767.

21. Miles, M.P., Andring, J.M., Pearson, S.D., Gordon, L.K., Kasper, C., Depner, C.M. and Kidd, J.R. (2008) Diurnal variation, response to eccentric exercise, and association of inflammatory mediators with muscle damage variables. $J$. Appl. Physiol., 104, 451-458.

22. Oosterom, D.L. and Betjes, M.G. (2006) Exertion-related abnormalities in the urine. Ned. Tijdschr. Geneeskd., 150, 606-610.

23. Chung, Y.L., Rider, L.G., Bell, J.D., Summers, R.M., Zemel, L.S., Rennebohm, R.M., Passo, M.H., Hicks, J., Miller, F.W. and Scott, D.L. (2005) Juvenile dermatomyositis disease activity collaborative study G. Muscle metabolites, detected in urine by proton spectroscopy, correlate with disease damage in juvenile idiopathic inflammatory myopathies. Arthritis Rheum., 53, 565-570.

24. Huerta-Alardin, A.L., Varon, J. and Marik, P.E. (2005) Bench-to-bedside review: rhabdomyolysis -- an overview for clinicians. Crit. Care, 9, 158-169.

25. Khan, F.Y. (2009) Rhabdomyolysis: a review of the literature. Neth. J. Med., 67, 272-283.

26. Duchen, M.R., Valdeolmillos, M., O’Neill, S.C. and Eisner, D.A. (1990) Effects of metabolic blockade on the regulation of intracellular calcium in dissociated mouse sensory neurones. J. Physiol., 424, 411-426.

27. Duncan, C.J. (1987) Role of calcium in triggering rapid ultrastructural damage in muscle: a study with chemically skinned fibres. J. Cell Sci., 87, 581-594.

28. Armstrong, R.B., Warren, G.L. and Warren, J.A. (1991) 
Mechanisms of exercise-induced muscle fibre injury. Sports Med., 12, 184-207.

29. Busch, W.A., Stromer, M.H., Goll, D.E. and Suzuki, A. (1972) $\mathrm{Ca}^{2+}$-specific removal of $\mathrm{Z}$ lines from rabbit skeletal muscle. J. Cell Biol., 52, 367-381.

30. Baird, M.F., Graham, S.M., Baker, J.S. and Bickerstaff, G.F. (2012) Creatine-kinase- and exercise-related muscle damage implications for muscle performance and recovery. $J$. Nutr. Metab., 2012, 960363.

31. Barding, G.A., Jr., Salditos, R. and Larive, C.K. (2012) Quantitative NMR for bioanalysis and metabolomics. Anal. Bioanal. Chem., 404, 1165-1179.

32. Jordan, K.W., Nordenstam, J., Lauwers, G.Y., Rothenberger, D.A., Alavi, K., Garwood, M. and Cheng, L.L. (2009) Metabolomic characterization of human rectal adenocarcinoma with intact tissue magnetic resonance spectroscopy. Dis. Colon. Rectum., 52, 520-525.

33. Ra, S.G., Maeda, S., Higashino, R., Imai, T. and Miyakawa, S. (2014) Metabolomics of salivary fatigue markers in soccer players after consecutive games. Appl. Physiol. Nutr. Metab., 39, 1120-1126.

34. Hicks, K.M., Onambele, G.L., Winwood, K. and Morse, C.I. (2016) Muscle damage following maximal eccentric knee extensions in males and females. PLOS ONE, 11, e0150848.

35. Newham, D.J., Jones, D.A. and Edwards, R.H. (1983) Large delayed plasma creatine kinase changes after stepping exercise. Muscle Nerve, 6, 380-385.

36. Vissing, K., Overgaard, K., Nedergaard, A., Fredsted, A. and Schjerling, P. (2008) Effects of concentric and repeated eccentric exercise on muscle damage and calpain-calpastatin gene expression in human skeletal muscle. Eur. J. Appl. Physiol., 103, 323-332.

37. Wilson, J.M., Kim, J.S., Lee, S.R., Rathmacher, J.A., Dalmau, B., Kingsley, J.D., Koch, H., Manninen, A.H., Saadat, R. and Panton, L.B. (2009) Acute and timing effects of betahydroxy-beta-methylbutyrate (HMB) on indirect markers of skeletal muscle damage. Nutr. Metab. (Lond), 6, 6 .

38. Baroni, B.M., Leal Junior, E.C., De Marchi, T., Lopes, A.L., Salvador, M. and Vaz, M.A. (2010) Low level laser therapy before eccentric exercise reduces muscle damage markers in humans. Eur. J. Appl. Physiol., 110, 789-796.

39. Selkow, N.M., Herman, D.C., Liu, Z., Hertel, J., Hart, J.M. and Saliba, S.A. (2015) Blood flow after exercise-induced muscle damage. J. Athl. Train, 50, 400-406.

40. Twist, C. and Eston, R.G. (2009) The effect of exerciseinduced muscle damage on perceived exertion and cycling endurance performance. Eur. J. Appl. Physiol., 105, 559567.

41. Chen, T.C., Lin, K.Y., Chen, H.L., Lin, M.J. and Nosaka, K. (2011) Comparison in eccentric exercise-induced muscle damage among four limb muscles. Eur. J. Appl. Physiol., 111, 211-223.

42. Penailillo, L., Blazevich, A., Numazawa, H. and Nosaka, K. (2015) Rate of force development as a measure of muscle damage. Scand. J. Med. Sci. Sports, 25, 417-427.

43. Clarkson, P.M. and Hubal, M.J. (2002) Exercise-induced muscle damage in humans. Am. J. Phys. Med. Rehabil., 81, S52-S69.

44. Rinard, J., Clarkson, P.M., Smith, L.L. and Grossman, M.
(2000) Response of males and females to high-force eccentric exercise. J. Sports Sci., 18, 229-236.

45. Ruoppolo, M., Scolamiero, E., Caterino, M., Mirisola, V., Franconi, F. and Campesi, I. (2015) Female and male human babies have distinct blood metabolomic patterns. Mol. Biosyst., 11, 2483-2492.

46. Tso, V.K., Sydora, B.C., Foshaug, R.R., Churchill, T.A., Doyle, J., Slupsky, C.M. and Fedorak, R.N. (2013) Metabolomic profiles are gender, disease and time specific in the interleukin-10 gene-deficient mouse model of inflammatory bowel disease. PLOS ONE, 8, e67654.

47. Dieli-Conwright, C.M., Spektor, T.M., Rice, J.C., Sattler, F.R. and Schroeder, E.T. (2009) Hormone therapy attenuates exercise-induced skeletal muscle damage in postmenopausal women. J. Appl. Physiol., 107, 853-858.

48. Saks, V. (2008) The phosphocreatine-creatine kinase system helps to shape muscle cells and keep them healthy and alive. J. Physiol., 586, 2817-2818.

49. Vigelso, A., Andersen, N.B. and Dela, F. (2014) The relationship between skeletal muscle mitochondrial citrate synthase activity and whole body oxygen uptake adaptations in response to exercise training. Int. J. Physiol. Pathophysiol. Pharmacol., 6, 84-101.

50. Koopman, R., Ly, C.H. and Ryall, J.G. (2014) A metabolic link to skeletal muscle wasting and regeneration. Front Physiol., 5, 32.

51. Powers, S.K. and Jackson, M.J. (2008) Exercise-induced oxidative stress: cellular mechanisms and impact on muscle force production. Physiol. Rev., 88, 1243-1276.

52. Reid, M.B., Shoji, T., Moody, M.R. and Entman, M.L. (1992) Reactive oxygen in skeletal muscle. II. Extracellular release of free radicals. J. Appl. Physiol., 73, 1805-1809.

53. Son, D.O., Satsu, H. and Shimizu, M. (2005) Histidine inhibits oxidative stress- and TNF-alpha-induced interleukin-8 secretion in intestinal epithelial cells. FEBS Lett., 579, 4671-4677.

54. Tidball, J.G. (2005) Inflammatory processes in muscle injury and repair. Am. J. Physiol. Regul. Integr. Comp. Physiol., 288, R345-R353.

55. Mangino, M.J., Murphy, M.K., Grabau, G.G. and Anderson, C.B. (1991) Protective effects of glycine during hypothermic renal ischemia-reperfusion injury. Am. J. Physiol., 261, F841-F848.

56. Rush, G.F. and Ponsler, G.D. (1991) Cephaloridine-induced biochemical changes and cytotoxicity in suspensions of rabbit isolated proximal tubules. Toxicol. Appl. Pharmacol., 109, 314-326.

57. Ascher, E., Hanson, J.N., Cheng, W., Hingorani, A. and Scheinman, M. (2001) Glycine preserves function and decreases necrosis in skeletal muscle undergoing ischemia and reperfusion injury. Surgery, 129, 231-235.

58. Ham, D.J., Murphy, K.T., Chee, A., Lynch, G.S. and Koopman, R. (2014) Glycine administration attenuates skeletal muscle wasting in a mouse model of cancer cachexia. Clin. Nutr., 33, 448-458.

59. Becker, A., Fritz-Wolf, K., Kabsch, W., Knappe, J., Schultz, S. and Volker Wagner, A.F. (1999) Structure and mechanism of the glycyl radical enzyme pyruvate formate-lyase. Nat. Struct. Biol., 6, 969-975. 
60. Dashko, S., Zhou, N., Compagno, C. and Piskur, J. (2014) Why, when, and how did yeast evolve alcoholic fermentation? FEMS Yeast Res., 14, 826-832.

61. Doi, Y. and Ikegami, Y. (2014) Pyruvate formate-lyase is essential for fumarate-independent anaerobic glycerol utilization in the Enterococcus faecalis strain W11. J. Bacteriol., 196, 2472-2480. 